\title{
Introduction to A Research Agenda for Academic Integrity: emerging issues in academic integrity research
}

Tracey Bretag

\section{Introduction}

Academic integrity underpins every aspect of education, from pre-school through to elementary school, secondary school, college, university and postgraduate research. Academic integrity is the cornerstone of ethical academic practice and is premised on a set of values most commonly described by the International Center for Academic Integrity (ICAI) as honesty, trust, fairness, respect and responsibility (ICAI, 1999 [2013]). Drawing on both the ICAI definition and the Exemplary Academic Integrity Project (2013), Universities Australia articulates the concept as part of the Academic Integrity Best Practice Principles:

Academic integrity means acting with the values of honesty, trust, fairness, respect and responsibility in learning, teaching and research. Universities consider that it is vital for students and all staff to act in an honest way and take responsibility for their actions and every part of their work. Staff should be role models to students. Academic integrity is important for an individual's and a university's reputation. (Universities Australia, 2017)

International research on academic integrity has been influenced by large-scale surveys of students' self-reported cheating in the United States (US) (McCabe, 1992; McCabe and Bowers, 1994; McCabe and Pavela, 1998; McCabe and Trevino, 1995, 1997), by technology-driven responses to plagiarism in the United Kingdom (UK) (Tennant et al., 2007), and by nationally funded research on academic integrity and contract cheating in Australia (Bretag et al., 2011, 2014, 2018a, 2018b). Extending the work of Australian and international 
researchers, my own work has conceptualized academic integrity as a complex, multi-stakeholder responsibility which goes well beyond students cheating or plagiarizing (Bretag, 2018). In company with other researchers (e.g., Carroll and Appleton, 2001; Bertram Gallant, 2008; HEA, 2011a, 2011b), I have consistently maintained that academic integrity is a teaching and learning issue; hence, we should foreground learning, rather than character. Having said that, providing students and staff with appropriate ethics training is an important part of promoting cultures of integrity. Recent threats to academic integrity, such as contract cheating (where students outsource their work to third parties), have focused attention on the role of governments, regulatory bodies and institutions to adequately resource teaching and provide critical support for vulnerable student cohorts. Other emerging themes in the field include how to engage students in academic integrity education, machine-based plagiarism, new technologies to authenticate student writing, and cybersecurity threats.

\section{A Research Agenda for Academic Integrity}

In keeping with the aims of the Elgar Research Agendas series, the purpose of A Research Agenda for Academic Integrity is to outline the future of research in academic integrity. Leading scholars have been given the opportunity to explore their subject in provocative ways, and to map out the potential directions of travel. Somewhat controversially, given the historical emphasis on individual students' self-reported cheating behaviours in the literature, this book starts with macro-level responsibilities. Irene Glendinning (Chapter 2) challenges us to consider the critical role played by external regulatory bodies in promoting and upholding academic integrity. Based on nearly a decade of research and consultancy, Glendinning demonstrates that a focus on individual students' motivations, values or character is somewhat disingenuous if quality assurance agencies are complacent or in denial about endemic corruption in the broader higher education context. Despite the fact that most quality assurance and accreditation bodies are aware of the symbiotic relationship between academic integrity and academic quality and standards, very few of these organizations make this relationship explicit, and even fewer provide effective responses when systemic breaches are uncovered.

Glendinning quite rightly reminds the reader that academic integrity is the responsibility of all members of the academic community; furthermore, it is not limited to student assessment, but applies to a broad range of higher education and research functions, including fraud in admissions and recruitment. She points out that one of the most serious threats to academic integrity is 
corruption within the quality assurance process itself, such as nepotism and cronyism, and lack of integrity within institutional governance. If external agencies include panel members who are open to bribery, or who are unconcerned about academic integrity, then the institutional evaluation process is seriously undermined, and opportunities for influencing or enhancing academic integrity across the higher education sector are reduced.

Moving from Glendinning's macro-view of corruption and academic integrity, authors from three regions where corruption is perceived to be pervasive, and academic integrity is a relatively new topic of inquiry, provide insights from their particular contexts. Pablo Ayala-Enríquez, Nathalia Franco-Pérez, Jean G. Guerrero-Dib and Gonzalo Pizarro-Puccio (Chapter 3) interrogate commonly held views about Latin American students being 'culturally programmed to cheat'. Based on their study of 1008 Colombian, Mexican and Chilean students, the authors found that despite having an in-depth knowledge of academic integrity principles and practices, Latin American students are unwilling to report others' dishonest behaviours and lack an understanding of the link between academic and social or professional integrity. The existence of a culture which focuses on high levels of success (such as a high Grade Point Average), in association with three additional factors - apathy, fear and solidarity - were found to influence Latin American students' propensity to cheat. Using the data from this study as a starting point, Ayala-Enríquez et al. invite future research which explores the disjuncture between Latin American students' ethical understandings and their behaviour, the avenues to encourage the reporting of academic dishonesty, and the relationships between academic integrity, civic culture and employment practices.

Tomáš Foltýnek and Dita Dlabolová (Chapter 4) examine the differences between Eastern Europe, other European countries, and English-speaking countries (USA, Canada, Australia, New Zealand and UK) and make the case that there are three primary factors which influence academic integrity practices: culture, material wealth and perceptions of corruption. The authors conclude that the academic integrity issues common to many Eastern European countries are the result of a lack of democracy in those contexts, underfunded education systems and systemic corruption. While much progress has been made in recent years through numerous European Union funded research projects, Foltýnek and Dlabolová call for academics to conduct more research into region-specific academic integrity issues, recognizing the cultural contexts of the numerous countries and institutions in the region. There is an urgent need for empirically based recommendations to meet the unique needs of the Eastern European higher education sector. 
There can be no doubt that there is a wealth of research, particularly work done since the late 1990s, which can provide the 'shoulders' upon which other regions, such as Latin America and Eastern Europe, may stand. In the UK, Australia and the USA, there is broad agreement that academic integrity is a teaching and learning issue, which simultaneously acknowledges the role of values and ethics training in academic integrity education. In 2008, Bertram Gallant challenged the US-centric view that cheating was a student problem which could be eliminated by 'fixing' the students, either through punishment or by developing their character. And then in 2017, Bertram Gallant suggested a range of practical ways to create a learning-oriented environment which made cheating the exception and learning the norm. In Chapter 5, Tricia Bertram Gallant returns to the two strands of this theme - leveraging cheating as a teachable moment - and asks: what now, and how? Building a theoretical framework based on the work of Tjosvold et al. (2004), Bertram Gallant suggests that:

if students are to learn from their academic integrity error, their experience after the error must include reflection, sharing experiences, examining unintended and undesired impacts of the error, analysing contributors to the error, and devising ways to correct the error and prevent it from happening again (Chapter 5, p. 60, this volume)

As the Director of Academic Integrity at the University of California (San Diego), Bertram Gallant draws on her extensive experience in developing seminar curriculum and establishing the Integrity Mentorship Program (IMP) and suggests a range of research questions which may be able to answer the question of 'what, if anything, can students learn through the process of being reported for cheating and through after-violation education such as a one-on-one mentoring programme or a structured curriculum delivered in a classroom-type setting?' (Chapter 5, p. 60, this volume). Bertram Gallant's chapter provides clear direction for future research on a topic which has preoccupied her for more than a decade. She just might be the most appropriate person to follow these lines of enquiry, and the longitudinal methodologies and ethnographic studies she recommends might provide an empirical base for the commonly held view that academic integrity and professional integrity are linked.

If there is consensus that academic integrity is a teaching and learning issue, why then are we so often angry when students breach integrity guidelines? Felicity Prentice (Chapter 6) addresses this question by returning to the early literature on plagiarism, often by scholars in composition and writing. Prentice notes the strength of emotions expressed by teaching staff when confronted 
with obvious plagiarism. However, Prentice also notes that while there have been hundreds of research papers on students' academic integrity experiences, motivations and understandings, there has been comparatively little research on educators' emotional responses to academic integrity breaches, and in particular their responses to the recent threat of contract cheating. Given the crucial role that academic staff have in deterring, detecting and responding to breaches, Prentice posits that investigating educators' emotions in the breach decision-making process is essential to understanding how to promote and uphold academic integrity:

While aspects of the role of the educator in the prevention, detection and management of contract cheating have been examined, there has not been a significant amount of research into the experiences, personal and professional, of educators when confronting contract cheating. (Chapter 6, p. 79, this volume)

Perhaps in an attempt to move away from regarding academic integrity as a moral issue worthy of strong emotions and strict admonitions, Amanda White (Chapter 7) shares an interactive approach to academic integrity education which literally makes learning about academic integrity 'fun'. Based on her own experience as an accounting academic trying to inculcate her students into the academic norms of the discipline, White developed a board game to teach students the concepts, rules and requirements of academic integrity. Her rationale for this approach was based on increasing awareness of the role that gamification can play at all levels of education, and a perusal of the literature which highlighted many interactive (mostly online) learning exercises to teach academic integrity, but only one published paper about an adapted 'Snakes and Ladders' board game. White makes the case that:

The importance of the social aspect and the need for a tactile experience ... may mean that in the area of academic integrity, playing games in person may be more impactful than playing games online. It can be posited that when playing a game in person, there is a greater opportunity for effective socialization and enculturation in the area of academic integrity. (Chapter 7, p. 90, this volume)

Having developed the 'Academic Integrity Board Game' and trialled it with hundreds of her own students, White provides a reflective assessment of its efficacy, and recommendations for further developments of the board game concept going forward. She calls for research which systemically evaluates the role of 'fun and games' as part of a multipronged approach to engaging and socializing students into the norms of academic integrity.

The theme of engagement is taken up by Kiata Rundle, Guy Curtis and Joseph Clare (Chapter 8), whose psychological research found that the number one 
reason that students report not cheating, is their engagement and commitment to learning. The vast majority of students do not outsource their work to third parties because they understand that doing so will undermine their own learning and ultimately negatively impact on their capabilities as professionals. The other reasons for not engaging in cheating, in descending order of influence, include morality and norms, fear of detection and punishment, self-efficacy and trust, and lack of opportunity. The authors outline a model based on the routine activity theory of crime 'to explain the circumstances that must be met, the characteristics of students to consider and the imperfect barriers that may prevent cheating; all of which must serendipitously align for contract cheating to occur' (Chapter 8, p. 108, this volume).

These findings add to the work of Bretag et al. (2018a), who found that the three factors which influence students' engagement in contract cheating were: (1) language other than English (LOTE) status; (2) the perception that there are 'lots of opportunities to cheat'; and (3) dissatisfaction with the teaching and learning environment. While Rundle et al. did not explore the role of language in their study, the two key factors influencing contract cheating behaviour, which both studies confirmed, related to opportunities to cheat and teaching and learning. Students who are motivated to learn, are appropriately socialized into the norms and values of the academic community, are confident in their own academic ability and would therefore not want or trust someone else to complete the work for them, and are not bombarded daily by marketing-savvy commercial services offering cheap and readily available 'assistance', are all less likely to engage in contract cheating. The research by Rundle et al. takes the field of academic integrity down a new, positive path - one which focuses our attention on what students are doing right, rather than what they are doing wrong - and this has implications for all aspects of academic and integrity-related research, regardless of stakeholder or subject.

Continuing the interest in contract cheating, Erica J. Morris (Chapter 9) provides a review of the contract cheating research, examines the way that responses to this phenomenon have changed over time, and identifies gaps in the research that need to be addressed. Morris provides a framework for addressing contract cheating based on the work of Bretag et al. (2018a), which relates to four key elements - institutional strategy and policy; academic practice; professional development; and understanding students - and uses this framework to summarize the research, most of which is relatively recent and dominated by Australian researchers. Morris concludes that the 'student voice' is not strong enough, and much more qualitative work needs to be undertaken 'to explore student attitudes, motivations and practices relating to good academic practice, and why and when students might use academic custom 
writing services' (Chapter 9, p. 122, this volume). Following on from Rundle et al.'s survey research, Morris calls for more in-depth studies involving students ... [to] shed further light on the reasons why the majority of students do not ever engage in contract cheating' (Chapter 9, p. 122 this volume). Certainly, the need to partner with students, and engage in qualitative work which goes beyond anonymous surveys, is an ongoing refrain throughout this volume.

Tracey Bretag and Rowena Harper (Chapter 10) expand on their preliminary work which showed that while students at non-university higher education providers (often referred to as colleges or independent higher education providers) outsource their work to third parties at comparable rates to their university peers ( 7 per cent versus 6 per cent, respectively), they are 12 times more likely to report using a professional service (Bretag et al., 2019). Bretag and Harper call for a much larger and more representative sample of students from a range of non-university higher education providers to be surveyed, along with educators from those same institutions. Echoing Morris, Bretag and Harper call for qualitative research to be undertaken to better understand the distinct challenges of students and educators in the college sector, drawing out the differences between the various types of higher education providers. The authors think that this is especially important in light of the critical role that many colleges play in preparing students for academic success at university. In addition, there is a need for longitudinal research which follows 'transfer' or 'pathway' students from college through to university completion, to determine whether there are any academic integrity issues unique to this cohort. Importantly, there should be opportunities for collaboration and sharing of good practice between colleges and universities, with each sector having something to offer the other, particularly around best practice in supporting vulnerable students.

Cath Ellis, Karen van Haeringen and David House (Chapter 11) take up another strand of the current preoccupation with contract cheating by focusing on institutional processes relating to the detection, reporting and investigation of contract cheating cases. It is evident that research which improves education providers' capacity to develop evidentiary foundations for fair and transparent investigations of contract cheating is becoming increasingly important in jurisdictions where contract cheating is illegal (for example, Australia, New Zealand, Ireland and 17 US states). Extending the conceptual framework proposed by Bretag and Mahmud (2016), Ellis et al. propose a set of evidentiary standards on which educational institutions can rely to determine that contract cheating has occurred. In addition, the authors propose a number of potential research directions, including: investigating how best to support students through the investigative process; developing evidence-based 
approaches to train and support academic staff to improve their ability to detect contract cheating; improving policy and procedural structures which encourage academic staff to report potential cheating; examining the key indicators both within and between documents which can be relied upon to substantiate contract cheating; and learning from substantiated allegations of contract cheating to inform subsequent investigations and ongoing quality assurance. The authors remind researchers of the need to 'retain a clear focus on solutions, while keeping procedural fairness and student welfare at the front of their minds' (Chapter 11, p. 148, this volume).

Contract cheating is clearly a global concern; however, most research to date has examined this phenomenon only in English-speaking countries. Sarah Elaine Eaton and Roswita Dressler (Chapter 12) set out to explore whether commercial academic writing services were operating in languages other than English. Their preliminary study, using a 'rapid review' process, found evidence of commercial operators offering academic work in Arabic, French, German, Hebrew, Italian, Latin, Mandarin, Portuguese, Spanish and Welsh. The authors' small study was one of the first to look at cheating services in languages other than English and they were shocked to find so little research which explored the scope of the market more fully. They call on multilingual researchers to take their small study as the impetus to systematically investigate commercial providers in educational contexts where English is not the medium of instruction. Without this research, the lessons from the extensive and recent international research on contract cheating will not be available, or may not seem relevant to regulatory bodies, institutions, educators and students in countries where languages other than English are spoken.

Ann M. Rogerson (Chapter 13) is also interested in language, but her curiosity relates to students' use of Internet-based paraphrasing tools, translation software and editing systems, and the way these tools may be used by students to breach academic integrity. Rogerson's preliminary research has indicated that use of these online tools is a type of outsourcing behaviour which is distinct from contract cheating, but nonetheless shares many of the same characteristics and motivations. The student who downloads a free essay from a file-sharing site, 'spins' it through a paraphrasing tool, or perhaps translates it into a different language and then back-translates it to the original language, as a means of generating a so-called 'original' document for submission, is involved in the same disengaged and transactional approach to learning that is evident in contract cheating. The only difference is that there is some machine-based interaction with the text, and perhaps the lack of payment as in cases where students contract out their work to commercial services. 
Rogerson notes the paucity of research on this topic, with the limited publications available tending to be the result of curiosity derived from observations of irregularities within an assessment task, rather than the result of a focused or systematic research agenda. While these small studies have added to anecdotal evidence and pointed to a potentially widespread problem, Rogerson maintains that there is a pressing need to explore who and how many people are actually using these tools, in addition to understanding why and how they are being used. Given the extensive literature on contract cheating which has indicated the strong influence of LOTE status, it might be surmised that use of these online tools is becoming widespread, not because students want to cheat (due to either individual or cultural norms) but rather because their linguistic ability is simply not of an appropriate standard. Finally, Rogerson suggests that we need to explore how the generated outputs are being used, and what happens with the materials uploaded to Internet-based tools for paraphrasing, proofreading and/or translation.

Katherine Seaton (Chapter 14) takes a completely new approach to academic integrity, a field traditionally dominated by concerns with textual transgression, and offers fresh insights based on her experience as a mathematics educator. With very little in the academic integrity literature on mathematics, or even the physical sciences or engineering, to guide her, Seaton sets out a research agenda for academic integrity in mathematics education. The author suggests that there are four main gaps which need to be addressed: policies and procedures (along with accompanying educational materials) which take into account the way that misconduct is manifested in mathematics; engagement with academic integrity research and scholarship by mathematics and statistics academics; collaboration between mathematics and non-mathematics scholars for mutual professional development; and discipline-specific induction for students into the norms of assessment in mathematics.

The author quite rightly recommends that approaches to promoting academic integrity in mathematics education need to be 'evidence-based, and not influenced by popular opinion, tradition, folklore or convenience' (Chapter 14, p. 179, this volume). Seaton points to the extensive body of work in computer science over the last 15 years as providing a suitable framework for gathering this evidence. This includes the use of both quantitative surveys to collect baseline data on the prevalence of misconduct, and qualitative methods to gain in-depth insights into the perceptions and practices of mathematics scholars, educators, academic support staff and students. Seaton concludes that 'the most useful outcomes of such investigations would be good-practice guides for task design and marking; advice for the wording of university or department policies in such a way as to respect diverse disciplinary needs; and educational 
materials for use by and with students of mathematics' (Chapter 14, p. 184, this volume).

Having traversed a wide array of emerging academic integrity topics, from the macro-level quality assurance to the micro-level integrity in mathematics education, this volume concludes with an all-encompassing threat and a call for urgent action. Phillip Dawson (Chapter 15) argues that while computer hacking and the need for cybersecurity has been an acknowledged area of concern for decades in other fields such as banking, healthcare and gambling, the relatively recent shift to online learning in higher education makes cybersecurity an area of critical concern for academic integrity. The limited literature to date has focused on identity verification and/or authorship verification and how these pose potential threats to academic integrity. It is clearly important to be able to verify that the students are who they say they are, at all stages of their learning journey, just as it is imperative that markers are able to determine who has produced a piece of work for assessment. Without these two authentications, the rationale for awarding grades and qualifications is rendered meaningless.

Dawson explores why academic integrity scholars appear not to have engaged with the cybersecurity literature and suggests that the 'adversarial' stance of cybersecurity - 'It does not focus on persuading or educating people to not hack; instead, it focuses on hardening computer systems against hacking' (Chapter 15, p. 192, this volume) - may seem to be at odds with the 'positive, preventive, educative approaches' (Chapter 15, p. 192, this volume) inherent to academic integrity scholarship and practice. Dawson therefore advocates for future collaboration between these two fields to meet the challenges of an increasingly digital education landscape. Dawson also suggests that priority should be given to the study of the student experience of technologies designed to safeguard integrity, but which may be perceived to be restrictive or invasive.

Taking a provocative stance - one which is likely to be met with some distaste and opposition by many scholars - Dawson calls for academic integrity researchers to adopt an adversarial approach which focuses much more explicitly on finding ways to stop students cheating. Dawson suggests that 'a fruitful research agenda for academic integrity involves thinking like both a hacker and a cheater' (Chapter 15, p. 195, this volume). As the final voice in this volume, Dawson's concern with mitigating potential disasters should not be seen as a return to the punitive, combative and hyperbolic stance of early academic integrity research. Rather, Dawson invites future research which draws on interdisciplinary and even discordant perspectives as a means of confronting and overcoming emerging threats to academic integrity. 
This book covers a vast terrain and invites both new and established academic integrity researchers to take up one or more of the themes identified, using a range of methodologies to investigate the perspectives of diverse stakeholders. It is anticipated that the research agendas for academic integrity in the not too distant future will look very different than the ones presented in this volume.

\section{References}

Bertram Gallant, T. (2008), Academic Integrity in the Twenty-First Century: A Teaching and Learning Imperative. San Francisco, CA: Jossey-Bass.

Bertram Gallant, T. (2017), 'Academic integrity as a teaching and learning issue: from theory to practice', Theory into Practice, 56 (2), 88-94. https://doi.org/10.1080/ 00405841.2017.1308173.

Bretag, T. (2018), 'Academic integrity', Oxford Research Encyclopedia of Business and Management. doi: 10.1093/acrefore/9780190224851.013.147.

Bretag, T., R. Harper, M. Burton, C. Ellis, P. Newton, P. Rozenberg, et al. (2018a), 'Contract cheating: a survey of Australian university students', Studies in Higher Education, 1-20. https://doi.org/10.1080/03075079.2018.1462788.

Bretag, T., R. Harper, M. Burton, C. Ellis, P.M. Newton, K. van Haeringen, et al. (2018b), 'Contract cheating and assessment design: exploring the relationship', Assessment and Evaluation in Higher Education. https://doi:10.1080/02602938.2018 .1527892 .

Bretag, T., Harper, R., Rundle, K., Newton, P., Ellis, C., Saddiqui, S. and van Haeringen, K. (2019), 'Contract cheating in Australian higher education: a comparison of non-university higher education providers and universities', Assessment and Evaluation in Higher Education, 45 (1), 125-139. http://dx.doi.org/10.1080/ 02602938.2019.1614146.

Bretag, T. and S. Mahmud (2016), 'A conceptual framework for implementing exemplary academic integrity policy in Australian higher education', in T. Bretag (ed.), Handbook of Academic Integrity, Singapore: Springer Science+Business Media, pp. 463-480. https://doi.org/10.1007/978-981-287-098-8_24.

Bretag, T., S. Mahmud, M. Wallace, R. Walker, C. James, M. Green, et al. (2011), 'Core elements of exemplary academic integrity policy in Australian higher education', International Journal for Educational Integrity, 7 (2), 3-12.

Bretag, T., S. Mahmud, M. Wallace, R. Walker, U. McGowan, J. East, et al. (2014), “"Teach us how to do it properly!" An Australian academic integrity student survey', Studies in Higher Education, 39 (7), 1150-1169. https://doi:10.1080/03075079.2013 .777406.

Carroll, J. and J. Appleton (2001), 'Plagiarism: a good practice guide', accessed 20 August 2019 at http://www.webarchive.org.uk/wayback/archive/20140614152728/ http://www.jisc.ac.uk/media/documents/programmes/plagiarism/brookes.pdf.

Exemplary Academic Integrity Project (2013), www.unisa.edu.au/EAIP.

Higher Education Academy (HEA) (2011a), 'Policy works: recommendations for reviewing policy to manage unacceptable academic practice in higher educa- 
tion', accessed 20 August 2019 at http://www.heacademy.ac.uk/resources/detail/ academicintegrity/policy_works.

Higher Education Academy (HEA) (2011b), 'Supporting academic integrity: approaches and resources for higher education', accessed 20 August 2019 at https://www .heacademy.ac.uk/system/files/supportingacademicintegrity_v2_0.pdfHEA 2011.

International Centre for Academic Integrity (ICAI) (1999 [2013]), 'Fundamental values project', accessed 20 August 2019 at http://www.academicintegrity.org/icai/ resources-2.php.

McCabe, D.L. (1992), 'The influence of situational ethics on cheating among college students', Sociological Inquiry, 62, 365-374.

McCabe, D.L. and W.J. Bowers (1994), 'Academic dishonesty among males in college: a thirty year perspective', Journal of College Student Development, 35 (1), 5-10.

McCabe, D.L. and G.R. Pavela (1998), 'The effect of institutional policies and procedures on academic integrity', in D. Burnett and L. Rudolph (eds), Academic Integrity vs. the Academic Dishonesty of College Students, Washington, DC: National Association of Student Personnel Administrators, pp. 75-94.

McCabe, D.L. and L.K. Trevino (1995), 'Cheating among business students: a challenge for business leaders and educators', Journal of Management Education, 19 (2), 205-218.

McCabe, D.L. and L.K. Trevino (1997), 'Individual and contextual influences on academic dishonesty: a multi-campus investigation', Research in Higher Education, 38, 379-396.

Tennant, P., G. Rowell and F. Duggan (2007), 'AMBeR Project', Joint Information Committee Systems (JISC), accessed 20 January 2018 at www.jiscpas.ac.uk.

Tjosvold, D., Z. Yu and C. Hui (2004), 'Team learning from mistakes: the contribution of cooperative goals and problem solving', Journal of Management Studies, 41 (7), 1223-1245.

Universities Australia (UA) (2017), 'Academic integrity best practice principles', accessed 20 August 2019 at https://www.universitiesaustralia.edu.au/Media-and -Events/Publications/UA-Academic-Integrity-Best-Practice-Principles. 